\title{
Evaluación poblacional y estrategia de monitoreo para Atelopus spurrelli en el Parque Nacional Natural Utría, Colombia
}

\author{
Population assessment and monitoring estrategy for \\ Atelopus spurrelli in the Utría National Natural Park, Colombia
}

\author{
Diego A. Gómez-Hoyos ${ }^{1}$, Wilmar Bolívar-G. ${ }^{2}$, \\ Carlos E. Burbano-Yandi², José Luis García ${ }^{3}$
}

\begin{abstract}
Resumen
Objetivos: Estimar la densidad poblacional de $A$. spurrelli mediante el método de muestreo por distancias y evaluar la potencia estadística con el diseño de muestreo implementado y las estimaciones de densidad obtenidas para un eventual monitoreo de la especie en el PNN Utría, Colombia. Metodología: La densidad se estimó con el método de muestreo por distancias en el software DISTANCE 6.0 Release 2, usando nueve transectos de longitud variable a lo largo de quebradas. Por su parte, la potencia estadística se estimó con el software Monitor versión 11.0. Resultados: Se obtuvo una densidad de 0,03 individuos $/ \mathrm{m}^{2}(\mathrm{CV}=12,8 \%)$ de $A$. spurrelli asociados con quebradas. La evaluación de la potencia estadística indicó que al momento de diseñar un programa de monitoreo para la especie implicaría dos muestreos anuales durante tres años para detectar cambios poblacionales exponenciales de hasta $12 \%$ con una potencia del $85 \%$. Conclusión: En un eventual programa de monitoreo para $A$. spurrelli debe evaluarse la capacidad operativa y técnica del personal a cargo, para decidir la periodicidad y frecuencia de los muestreos teniendo en cuenta los resultados obtenidos en el presente estudio.
\end{abstract}

Palabras clave: Amphibia, Estimación poblacional, Sapo arlequín.

\begin{abstract}
Objectives: Estimate $A$. spurrelli density by distance sampling and perform a statistical power analysis for an eventual monitoring, based on the sampling and data obtained in the Utría NNP. Methodology: Population density was estimated by distance sampling with the software DISTANCE 6.0 Release 2, using nine transects following the course of the streams with variable length. The statistical power was estimated with the software Monitor 11.0. Results: The estimated density was 0,03 individuals $/ \mathrm{m}^{2}$ (CV $=12,8 \%)$ of $A$. spurrelli associated to streams. The statistical power showed that implementing a monitoring program for the species would imply two annual samplings for three years, in order to detect exponential changes up to $12 \%$ with a power of $85 \%$. Conclusion: In an eventual monitoring program for $A$. spurrelli, operational and technical capacity from staff in charge must be assessed, in order to determine sampling periodicity and frequency according to the results obtained in this study.
\end{abstract}

Keywords: Amphibia, Harlequin toad, Population estimation.

\section{Introducción}

El género Atelopus es uno de los grupos más diversos de la familia Bufonidae y comprende 96 especies reconocidas hasta el momento (Frost 2013). Su distribución se restringe a la región Neotropical,

\footnotetext{
Grupo de Investigación y Asesoría en Estadistica, Universidad del Quindío, Armenia, Colombia. e-mail: dagomezh@uqvirtual.edu.co

2 Grupo de Investigación en Ecología Animal, Universidad del Valle, Departamento de Biología, Cali, Colombia. e-mail: wilmar.bolivar@correounivalle.edu.co

3 Parques Nacionales Naturales de Colombia - Territorial Pacífico, Cali, Colombia. e-mail: pacificoinvestigacionespnn@gmail.com Fecha recepción: Noviembre 26, 2013 
presentando la mayor riqueza en los Andes, entre 1500 y 2000 m de elevación (Lötters 2007). La mayor diversidad se encuentra en Colombia, que alberga 43 especies, seguido por Ecuador, Perú y Venezuela. El género tiene menor representación en las regiones Amazónica y Guayanesa, así como en Centroamérica.

Los Atelopus, comúnmente conocidos como sapos arlequines, comprenden un grupo altamente vulnerable a la extinción debido a sus endemismos locales (La Marca et al. 2005, Luger et al. 2008). De acuerdo con la UICN, hasta ahora tres especies han sido reportadas extintas (EX) y 74 se encuentran categorizadas en peligro crítico (CR), de las cuales se cree que una importante proporción esté extinta (Lötters 2007). En general, Lötters et al. (2004) reconocen que muchos científicos y conservacionistas convergen en que los sapos arlequines son difíciles de observar o no han sido registrados recientemente. Por lo anterior, es posible que este género rico en especies esté diezmando y pueda eventualmente desaparecer (Lötters et al. 2004, Lötters 2007).

En Colombia el problema es aún más crítico, porque cerca del $80 \%$ de los sapos arlequines se encuentran amenazados de extinción (base de datos UICN 2013). De hecho, reportes anecdóticos de herpetólogos colombianos, indican que las poblaciones de Atelopus distribuidas en los Andes (por encima de 1500 metros de elevación) no han vuelto a observarse y podrían estar extintas, sin conocerse para muchas especies aspectos de su biología, historia natural y, peor aún, las causas de su desaparición. Pese a este panorama, algunas poblaciones han sido registradas tanto en las tierras bajas del Pacífico y Caribe colombiano, como en la Sierra Nevada de Santa Marta (Rueda 2008), representando esto una oportunidad para conocer y conservar las especies de Atelopus que aún persisten.

En el Parque Nacional Natural (PNN) Utría ha sido registrada recientemente una población de Atelopus spurrelli. Esta especie es conocida comunmente como sapo arlequín del Chocó y se distribuye en las tierras bajas del Pacífico colombiano (departamentos de Valle del Cauca, Risaralda y Chocó), en un rango altitudinal entre 50 y $500 \mathrm{~m}$ de elevación, en la selva húmeda tropical (Bolívar y Lötters 2004, Rivera 2005). La especie suele observarse en la hojarasca, rocas de quebradas o sobre la vegetación has- ta casi un metro de altura, generalmente asociada con cuerpos de agua; es una especie diurna, pero suele encontrarse en la noche sobre plantas cerca de las quebradas. Aunque se desconocen muchos aspectos de su biología e historia natural, los arlequines suelen seguir las generalizaciones del género: amplexus prolongados, huevos acuáticos adheridos a hojas o rocas en las quebradas, y renacuajos que se desarrollan en los remansos de agua (Lötters 1996, Lötters 2007).

El sapo arlequín del Chocó se encuentra categorizado actualmente como vulnerable (VU), de acuerdo con la lista roja de la UICN, por ser especialmente susceptible a presentar disminuciones poblacionales a causa de la acción del hongo patogénico Batrachochytrium dendrobatidis (Bolívar y Lötters 2004). Otras amenazas que enfrenta la especie son la expansión de la frontera agrícola, los cultivos ilícitos y el tráfico ilegal, que ha sido reportado en la literatura (La Marca et al. 2005). A pesar de su vulnerabilidad a la extinción, no se conocen estudios poblacionales de línea base que sirvan para diseñar e implementar programas de monitoreo.

Ante la crisis que enfrentan actualmente los sapos arlequines, se han propuesto varias medidas de mitigación, entre las que se resalta el trabajo de campo extensivo para evaluar el estado de las poblaciones e iniciar programas de monitoreo y manejo (La Marca et al. 2005, Lötters 2007, Lampo et al. 2012). Por esta razón, es necesario iniciar estudios de línea base que permitan evaluar las poblaciones y plantear planes de monitoreo a largo plazo, que permitan tomar medidas de manejo que eviten la extinción de poblaciones o especies. Sin embargo, es necesario discriminar las fluctuaciones poblacionales naturales de las disminuciones causadas por factores de amenaza, porque los programas de monitoreo deben evitar medidas innecesarias de conservación y manejo (Gardner 2001).

En este estudio se presenta la evaluación de línea base de una población de A. spurrelli del PNN Utría y se analiza la potencia estadística para detectar cambios poblacionales con el fin de proponer un diseño de monitoreo para esta especie.

\section{Metodología}

Área de estudio. El PNN Utría se encuentra 
ubicado en el Pacífico colombiano, departamento del Chocó, en jurisdicción de los municipios de Bahía Solano, Nuquí, Alto Baudó y Bojayá (BirdLife International 2012). El Parque se ubica entre $05^{\circ} 52^{\prime}$ - $06^{\circ} 09^{\prime} \mathrm{N}$ y $077^{\circ} 08^{\prime}-077^{\circ} 24^{\prime} \mathrm{W}$ y presenta un gradiente de elevación desde $0 \mathrm{~m}$ hasta $1140 \mathrm{~m}$, cubriendo un área total de 77.750 ha de las cuales aproximadamente 59.000 son terrestres (Vásquez y Serrano 2009).

El estudio se llevó a cabo en la Ensenada de Utría (Figura 1) que corresponde a una zona particular $\left(06^{\circ} 0.43^{\prime} \mathrm{N}\right.$ y $\left.077^{\circ} 16.27^{\prime} \mathrm{W}\right)$ del PNN Utría porque hace parte del complejo de la Serranía del Baudó y del litoral, presentando una «laguna» de agua marina rodeada por playas, manglares y montañas de poca elevación, con una longitud aproximada de $6 \mathrm{~km}$ (Vásquez y Serrano 2009). Esta zona presenta una temperatura que oscila entre $27^{\circ} \mathrm{C}$ y $30^{\circ} \mathrm{C}$, con humedad relativa de $90 \%$ y una precipitación promedio multianual de $10.000 \mathrm{~mm}$, que corresponde a formaciones vegetales de selva húmeda tropical (Vásquez y Serrano 2009).

Diseño de muestreo. Entre los días 20 y 26 de febrero de 2012 se realizaron inspecciones a lo largo de transectos, siguiendo el curso de quebradas, en busca de individuos de A. spurrelli. Se recorrieron un total de nueve transectos con longitud variable (Tabla 1), los cuales fueron medidos con un GPS (máximo $6 \mathrm{~m}$ de error). Los recorridos a lo largo de las quebradas se realizaron entre las 08:00 y 11:00

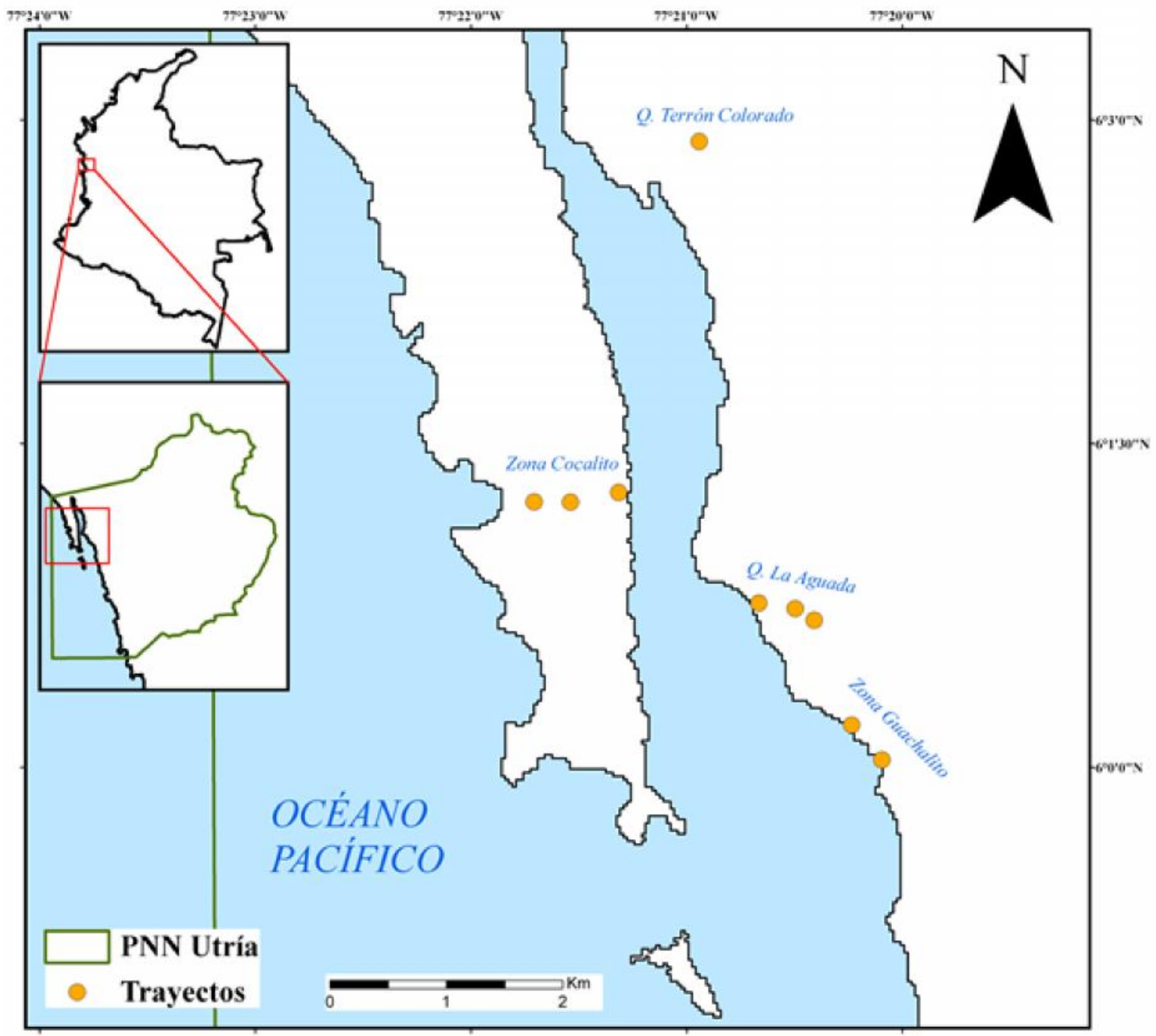

Figura 1. Ubicación Parque Nacional Natural Utría, Colombia. 
Tabla 1. Quebradas inspeccionadas en el PNN Utría, longitud de transectos y esfuerzo de muestreo aplicado.

\begin{tabular}{lccc}
\hline Quebrada & Transecto & Longitud $(\mathbf{m})$ & Esfuerzo de muestreo (horas/persona) \\
\hline La Aguada & 1 & 152 & 3,5 \\
& 2 & 136 & 3,3 \\
Cocalito 1 & 3 & 133 & 2,2 \\
Cocalito 2 & 4 & 130 & 3,2 \\
& 5 & 156 & 2,9 \\
Terrón Colorado & 6 & 125 & 2,3 \\
Guachalito & 7 & 117 & 1,6 \\
& 8 & 110 & 2,5 \\
Promedio & 9 & 142 & 2,1 \\
Desviación estándar & & 133,44 & 2,62 \\
\hline
\end{tabular}

horas, y entre las 14:00 y 17:00 horas. Los individuos observados durante los recorridos fueron mantenidos individualmente en bolsas de plástico donde se les midió en la longitud rostro-cloacal usando un calibrador con precisión $0,1 \mathrm{~mm}$. Este procedimiento evita el contacto de la herramienta con los individuos y ayuda a minimizar el riesgo de contaminación con patógenos. Adicionalmente, se registró la distancia perpendicular desde el punto en que se encontró el individuo hasta la orilla de la quebrada, porque este es el espacio efectivo para el desplazamiento y organización espacial de los sapos.

Muestreo por distancias. Se estimó la densidad poblacional de la especie a través del método de muestreo por distancias perpendiculares en transectos, por ser una técnica ampliamente usada para estimar la densidad de poblaciones biológicas (Buckland et al. 1993, Thomas et al. 2010). Para obtener estimaciones confiables de densidad, Buckland et al. (1993) recomiendan mínimo entre 60 y 80 observaciones, así como medidas de distancia perpendicular tomadas con exactitud. Este método basado en modelos ofrece menos sesgo que los producidos cuando se asume detectabilidad perfecta (Barry y Welsh 2001) como con métodos basados en índices de abundancia o densidad, que deben ser tratados con precaución debido a la variabilidad temporal en la tasa de captura de los Atelopus (Lampo et al. 2012).

El muestreo por distancias consiste en realizar recorridos en una línea de transecto y contar los animales observados, midiendo la distancia entre el transecto y el sitio donde se observó el animal. Con este método se obtuvo la distribución de las distancias y con ayuda del programa DISTANCE 6.0
Release 2 (Thomas et al. 2010) se generaron seis modelos basados en tres funciones en combinación con tres series de expansión, de acuerdo con las recomendaciones de Buckland et al. (2001): Halfnormal (Cosine y Hermite polynomial), Uniform (Cosine y Simple polynomial) y Hazard rate (Cosine y Simple polynomial). El modelo que mejor se ajustó a la distribución de las distancias perpendiculares se eligió bajo el criterio de información de Akaike (CIA), donde los menores valores del CIA indican el modelo más verosímil (Burnham y Anderson 2002). El modelo elegido se utilizó para estimar la densidad y probabilidad de detección de A. spurrelli.

Análisis de potencia. Se propuso un diseño de evaluación poblacional para mantener vigilada la densidad de A. spurrelli, en caso de identificar necesidades de manejo inmediatas (que no son producto de un programa de monitoreo establecido) para evitar su extinción local. Para esto se realizaron análisis de potencia usando el programa MONITOR versión 11.0 (Gibbs y Ene 2010) para estimar la potencia estadística de detectar fluctuaciones en la densidad poblacional, usando el método de muestreo por distancias. El programa MONITOR usa simulaciones de Monte Carlo para estimar la potencia de un programa de monitoreo, a partir de variables relacionadas con el diseño de los muestreos (Gibbs y Ene 2010). Así, se determinó la sensibilidad del método utilizado para identificar cambios poblacionales con al menos $80 \%$ de potencia con eventuales monitoreos de tres a cinco años y una frecuencia de uno a cuatro muestreos anuales. De esta manera se estimó la magnitud de las fluctuaciones anuales que pueden identificarse en la población con un diseño de muestreo específico. Los análisis se realizaron con una significancia de 
$5 \%(\alpha=0,05)$ con una potencia deseada para detectar la disminución de 0,8 , y un $\alpha=0,1$ con una potencia de 0,9 , que constituyen valores tradicionales para estos análisis (Schwarz 2013).

\section{Resultados}

Se observaron 150 individuos de A. spurrelli incluyendo postmetamorfos, juveniles, subadultos y adultos (hembras y machos, Figura 2). La falta de claridad en la diferenciación de los machos y las hembras no permitió discriminar por categorías de sexo, aunque se espera que las hembras se encuentren en el rango de longitud mayor (26-31 mm). No se encontraron huevos ni renacuajos, pero si individuos de talla pequeña y cuatro parejas amplectantes (Figura 3 ), lo que indica actividad reproductiva cercana a las fechas de muestreos.

Muestreo por distancias. La función Half normal con ambas series de expansión (Cosine y

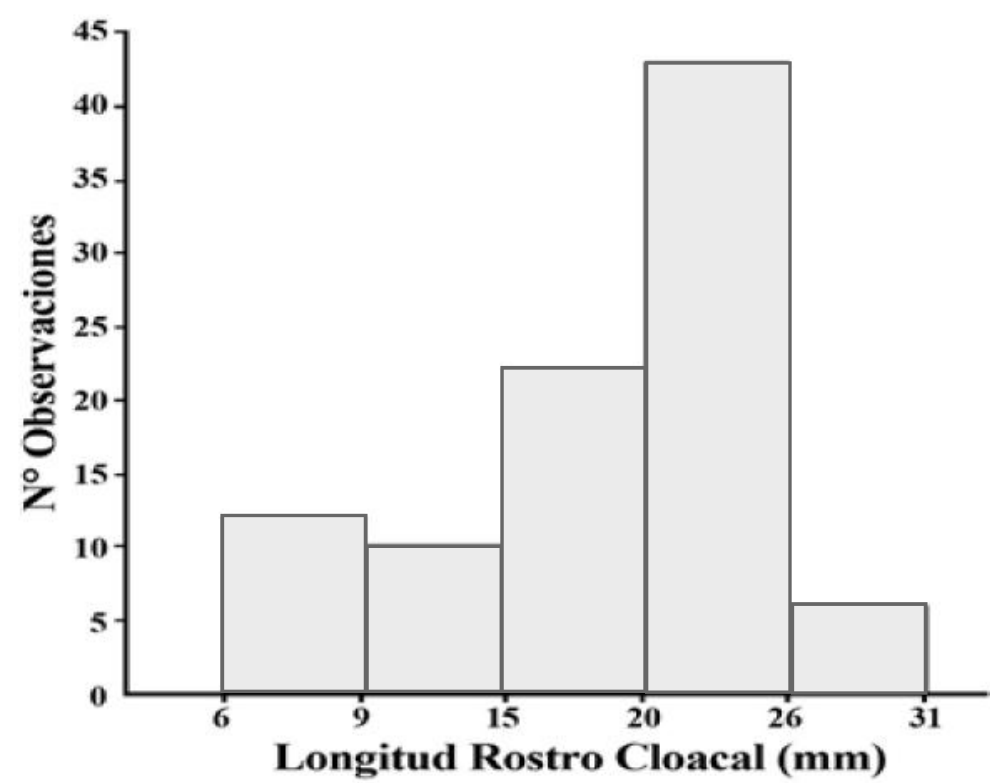

Figura 2. Distribución de frecuencias de la longitud rostro cloacal en los individuos de $A$. spurrelli observados en quebradas del PNN Utría. Los rangos de longitud pretenden dar una aproximación a las clases de edad en la especie (postmetamorfos: 6-9 mm; juveniles: $9-15 \mathrm{~mm}$; subadultos: $15-20 \mathrm{~mm}$; adultos: $20-31 \mathrm{~mm}$ ).

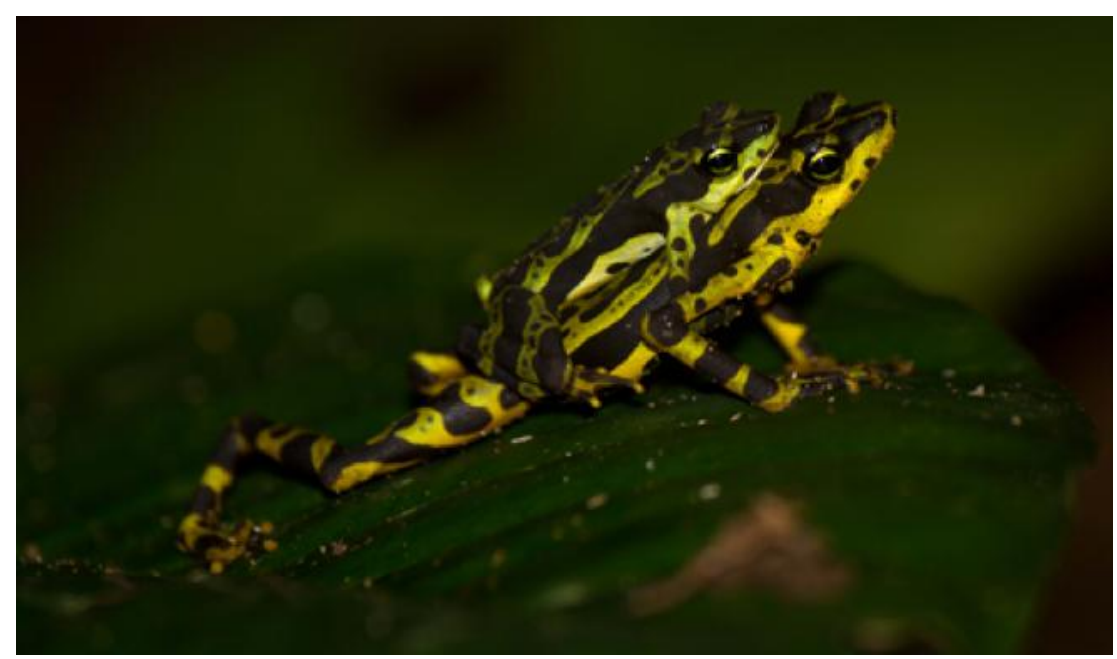

Figura 3. Pareja amplectante de $A$. spurrelli, no capturada, en el Parque Nacional Natural Utría, Colombia (foto: Diego Gómez Hoyos). 
Tabla 2. Modelos generados y estimación de la densidad de A. spurrellien el Parque Nacional Natural Utría.

\begin{tabular}{llcccc}
\hline Función & \multicolumn{1}{c}{ Serie } & CIA & CIA $\Delta$ & Parámetros & \multicolumn{2}{c}{ Densidad (IC 95\%) } \\
\hline Half Normal & Hermite polynomial & 358,53 & 0 & 1 & $0,029(0,022-0,038)$ \\
& Cosine & 358,53 & 0 & 1 & $0,029(0,022-0,038)$ \\
Uniform & Cosine & 359,84 & 1,32 & 2 & $0,029(0,022-0,039)$ \\
Hazard Rate & Cosine & 359,89 & 1,36 & 2 & $0,029(0,021-0,039)$ \\
& Simple polynomial & 359,89 & 1,36 & 2 & $0,029(0,021-0,039)$ \\
\hline
\end{tabular}

Tabla 3. Estimaciones de la potencia estadística y la disminución exponencial anual de la población con diseños de monitoreo de 3 y 5 años y eventos de muestreo entre 1 y 4 anuales.

\begin{tabular}{|c|c|c|c|c|c|}
\hline \multirow{2}{*}{$\begin{array}{l}\text { Años de } \\
\text { seguimiento }\end{array}$} & \multirow{2}{*}{$\begin{array}{l}\text { Muestreos } \\
\text { por año }\end{array}$} & \multirow{2}{*}{$\begin{array}{l}\text { Significancia } \\
(\alpha)\end{array}$} & \multicolumn{2}{|c|}{ Potencia } & \multirow{2}{*}{$\begin{array}{c}\text { Cambio exponencial } \\
\text { anual (\%) }\end{array}$} \\
\hline & & & deseada & $(1-\beta)$ & \\
\hline \multirow[t]{8}{*}{3} & 1 & 0,05 & 0,8 & 0,441 & 20 \\
\hline & & 0,1 & 0,9 & 0,717 & 20 \\
\hline & 2 & 0,05 & 0,8 & 0,867 & 14 \\
\hline & & 0,1 & 0,9 & 0,847 & 12 \\
\hline & 3 & 0,05 & 0,8 & 0,929 & 12 \\
\hline & & 0,1 & 0,9 & 0,907 & 10 \\
\hline & 4 & 0,05 & 0,8 & 0,905 & 10 \\
\hline & & 0,1 & 0,9 & 0,874 & 8 \\
\hline \multirow[t]{8}{*}{5} & 1 & 0,05 & 0,8 & 0,841 & 12 \\
\hline & & 0,1 & 0,9 & 0,885 & 10 \\
\hline & 2 & 0,05 & 0,8 & 0,928 & 8 \\
\hline & & 0,1 & 0,9 & 0,841 & 6 \\
\hline & 3 & 0,05 & 0,8 & 0,915 & 6 \\
\hline & & 0,1 & 0,9 & 0,956 & 6 \\
\hline & 4 & 0,05 & 0,8 & 0,971 & 6 \\
\hline & & 0,1 & 0,9 & 0,844 & 4 \\
\hline
\end{tabular}

Hermite polynomial) fueron los modelos que mejor se ajustaron a la distribución de las distancias perpendiculares registradas, con los menores valores del CIA (Tabla 2). A pesar de que los modelos generados no muestran una clara diferenciación en su ajuste, la estimación de los parámetros fue similar con cada uno de ellos (Tabla 2). Con estos modelos la densidad poblacional estimada para $A$. spurrelli fue de 0,03 individuos $/ \mathrm{m}^{2}$ (IC $95 \%=0,022-0,038$; $\mathrm{CV}=12,8 \%$ ) con una probabilidad de detección de $42,1 \%$ (IC $95 \%=37,49-47,19 ; \mathrm{CV}=5,8 \%$ ) y una amplitud de banda efectiva de 2,08 m (IC 95\%=1,862,$34 ; \mathrm{CV}=5,8 \%$ ). El componente que más aportó a la varianza de la densidad fue la tasa de encuentro con $79,4 \%$.

Análisis de potencia. La potencia estadística para detectar disminuciones en la densidad poblacional de A. spurrelli, depende del tiempo definido para realizar las evaluaciones de la población y del número de muestreos que se realizarían anualmente (Tabla 3). En general, la potencia fue más alta y sensible para identificar disminuciones en cinco años de evaluaciones y hasta con dos muestreos por año. Sin embargo, recomendamos diseñar un programa de seguimiento poblacional que implique dos muestreos anuales para un programa de evaluación de tres años que detectaría disminuciones exponenciales de hasta $12 \%$ anual con una potencia cercana a $85 \%$ (Tabla $3)$. Esta evaluación poblacional podría detectar aumentos de la misma magnitud y potencia, porque puede aumentar la densidad de sapos asociados con las quebradas (Tabla 3). Los aumentos eventuales de la densidad de sapos en las quebradas se presentaría potencialmente por eventos reproductivos, en el que se espera que los individuos adultos que se encuentran al interior del bosque (que no fueron registrados en las quebradas) se acerquen a los cuerpos de agua. 


\section{Discusión}

La frecuencia de las categorías de edad observadas para A. spurrelli muestran un patrón recurrente de algunas especies del género. Los individuos sexualmente inmaduros y potenciales machos se encuentran cerca a los cuerpos de agua (Figura 2), mientras que las hembras se desplazan hacia las quebradas sólo cuando están listas para reproducirse (Lötters 1996, Lötters 2007). Aunque no hay certeza de las épocas reproductivas de la especie ni su relación con parámetros ambientales, en el presente estudio se evidenció actividad reproductiva reciente por la presencia de postmetamorfos y parejas amplectantes.

El modelo para la estimación de los parámetros poblacionales estuvo representado por la función Half normal y la serie Hermite polynomial. Esta función es conveniente porque los histogramas de datos de distancia usualmente disminuyen con el aumento en distancia al transecto, y es sugerida junto a Hermite polynomial cuando los datos no han sido truncados (Buckland et al. 1993), como es el caso de los registros actuales. Además, este modelo presentó valores de 0 en CIA, lo que indica que es el mejor modelo generado (Thomas et al. 2010). Sin embargo, los modelos en general no mostraron una clara diferenciación en su ajuste, sin ser esto inconveniente porque la estimación de parámetros no difiere entre ellos (Tabla 2) (Buckland et al. 1993).

Infortunadamente, no se conocen estudios que reporten la densidad poblacional de A. spurrelli, por lo tanto, no es posible realizar comparaciones para identificar el estado de la población respecto a una estimación previa. Para otras especies de Atelopus se dispone de estimaciones de densidad a través de índices, por lo tanto no se pueden realizar comparaciones directamente. Sin embargo, la densidad de $A$. spurrelli parece más baja que las estimadas para $A$. ignescens $\left(0,222-0,585\right.$ individuos $\left./ \mathrm{m}^{2}\right)$ en el páramo del Antisana en Ecuador y A. varius (0,065-0,755 individuos $/ \mathrm{m}^{2}$ ) en la cabecera del río Lagarto en Costa Rica (datos disponibles en La Marca et al. 2005). Respecto a la densidad de A. hoogmoedi $(0,001-$ 0,013 individuos $/ \mathrm{m}^{2}$ ) en el Parque Natural Brownsberg en Suriname (Luger et al. 2008), la densidad del sapo arlequín del Chocó es aparentemente mayor.
De acuerdo con Bolívar y Lötters (2004), las poblaciones de A. spurrelli no muestran disminuciones dramáticas de sus poblaciones. Sin embargo, Castro-Herrera y Bolívar-García (2010) indican que las poblaciones en las quebradas del litoral Pacífico del Valle del Cauca han ido desapareciendo. Excepto por estos precedentes anecdóticos, las poblaciones de $A$. spurrelli no se han evaluado con metodologías que permitan estimaciones confiables, lo que imposibilita tomar medidas de conservación y manejo adecuadas, basadas en la detección de cambios en las poblaciones causadas por factores de amenaza. Por esta razón, la estimación actual de densidad para $A$. spurrelli es la línea base para iniciar evaluaciones y/ o monitoreos en el PNN Utría, que permitirán diferenciar las fluctuaciones naturales de sus poblaciones de las disminuciones causadas por factores de amenaza.

Los programas de monitoreo o el diseño de evaluaciones poblacionales debe ser lo más eficiente posible (e.g. capacidad para detectar disminuciones a tiempo para tomar medidas de manejo), con el uso de la menor cantidad de recursos disponibles (personal de campo, presupuesto, etc.). En el caso de los anfibios que presentan disminuciones poblacionales drásticas y en poco tiempo (Lips 1999, Young et al. 2001) debe procurarse obtener la mayor sensibilidad para detectar disminuciones en el menor tiempo posible. Teniendo en cuenta esto, presentamos la potencia estadística de detectar cambios en la densidad poblacional de A. spurrelli (Tabla 3) con el diseño de muestreo implementado en el presente estudio para decidir la frecuencia y periodicidad en un eventual programa de monitoreo de la especie en el PNN Utría de acuerdo con las posibilidades técnicas y operativas.

\section{Conclusiones}

Teniendo en cuenta la crisis actual que enfrentan los representantes del género Atelopus, es indispensable iniciar seguimientos poblacionales que permitan identificar las fluctuaciones naturales de las causadas por factores de amenaza. Por esta razón, se deben establecer umbrales de disminución poblacional como referencia para tomar decisiones de manejo fundamentadas y a tiempo. Las estimaciones de parámetros poblacionales a partir de modelos son nece- 
sarias para realizar estos seguimientos, pues los métodos basados en índices de abundancia sólo podrán compararse espacial y temporalmente si se asegura detectabilidad perfecta o se conoce que es proporcional a la verdadera abundancia, supuestos difíciles de cumplir.

En un eventual programa de monitoreo de $A$. spurrelli en el PNN Utría, se debe evaluar la capacidad operativa y técnica del personal para decidir la periodicidad y frecuencia de los muestreos. Si bien se presenta un resumen de la potencia de detectar cambios poblacionales en escenarios de monitoreo a corto plazo, el programa debe ser adaptado al diseño de muestreo que realmente se implemente, los resultados que se obtengan y la experiencia adquirida en el proceso, de esta manera podría diseñarse un programa de monitoreo efectivo. También es necesario incluir la medición de variables que puedan ayudar a explicar las variaciones poblacionales que se registren. Vale la pena aclarar que la rigurosidad del programa de monitoreo, aunque indispensable no debe ser estricta en caso de detectar disminuciones drásticas como ha ocurrido con algunas poblaciones de anfibios, de ser necesario deben tomarse decisiones con la evidencia disponible hasta el momento buscando la menor incertidumbre posible.

El presente estudio se restringió en las quebradas y tuvo poca representatividad temporal en los muestreos, lo que implica que durante el seguimiento de la población se adapten aspectos metodológicos conforme se obtienen datos poblacionales y experiencia en los muestreos. Pese a esto, los insumos aportados en este estudio son indispensables para diseñar e iniciar un programa de monitoreo para el sapo arlequín del Chocó.

\section{Agradecimientos}

Este proyecto fue realizado gracias al apoyo económico de Parques Nacionales Naturales de Colombia, a través del fondo de investigaciones «Mono Hernández» código 225. Agradecemos al personal del Parque Nacional Natural Utría y a la comunidad de El Valle (Bahía Solano), quienes nos apoyaron y participaron durante las actividades del proyecto. $\mathrm{Al}$ equipo del programa Colombia de WCS por su apoyo y asesoría durante el desarrollo de este estudio, así como a Ángela María González Colorado por el apoyo en la elaboración de la cartografía. A los revisores anónimos y a Tatiana Suárez por sus aportes para mejorar el manuscrito.

\section{Literatura citada}

Barry SC, Welsh AH. 2001. Distance sampling methodology. $J$ $R$ Stat Soc. 63 (Part 1): 31-53.

BirdLife International. Important bird areas factsheet: Parque Nacional Natural Ensenada de Utría. (En línea) 2012 (Acceso 24 marzo) URL disponible en: http:// www.birdlife.org

Bolívar W, Lötters S. Atelopus spurrelli. (En línea) 2004 (Acceso 24 marzo) URL disponible en: http://www.iucnredlist.org

Buckland ST, Anderson DR, Burnham KP, Laake JL. 1993. Distance sampling: Estimating abundance of biological populations. London: Chapman and Hall. 446 pp.

Buckland ST, Anderson DR, Burnham KP, Laake JL, Borchers D, Thomas L. 2001. Introduction to the distance sampling: estimating abundance of biological popu-lations. Oxford: Oxford University Press.

Burnham KP, Anderson DR. 2002. Model selection and multimodel inference: A practical information-theoretic approach. 2nd ed. New York: Springer-Verlag. 488 pp.

Castro-Herrera F, Bolívar-García W. 2010. Libro Rojo de los Anfibios del Valle del Cauca. Cali: casa editorial, no la impreta. $200 \mathrm{pp}$.

Frost, DR. Amphibian species of the World: an online reference. Version 5.6 (9 January 2013). (En línea) 2013 (Acceso 24 noviembre) URL disponible en: http://research.amnh. org/ herpetology/amphibia/index.html. New York: American Museum of Natural History.

Gardner T. 2001. Declining amphibian populations: a global phenomenon in conservation biology. An Biodivers Conservat. 24 (2): 25-44.

Gibbs JP, Ene E. Program monitor: Estimating the statistical power of ecological monitoring programs. Version 11.0.0 (En línea) 2010 (Acceso 25 mayo). URL disponible en: www.esf.edu/efb/gibbs/monitor/

La Marca E, Lips KR, Lötters S, Puschendorf R, Ibáñez R, Rueda-Almonacid JV, 2005. Catastrophic population declines and extinctions in neotropical harlequin frogs (Bufonidae: Atelopus). Biotropica 37 (2): 190-201.

Lampo M, Celsa SJ, Rodríguez-Contreras A, Rojas-Runjaic F, García CZ. 2012. High turnover rates in remnant populations of the harlequin frog Atelopus cruciger (Bufonidae): Low risk of extinction? Biotropica. 44 (33): 420-6.

Lips KR. 1999. Mass mortality and population declines of anurans at an upland site in Western Panama. Conserv Biol. 13 (1): 117-25.

Lötters S. 1996. The neotropical toad genus Atelopus. Checklist - Biology - Distribution. Köln: M Vences \& Glaw Verlags.

Lötters S, La Marca E, Stuart S, Gagliardo R, Veith M. 2004. A new dimension of current biodiversity loss. [Revista en línea]. Herpetotropicos. 1(3): 29-31. (Acceso 20 mayo de 2012). URL disponible en: http://erevistas.saber. ula.ve/ index.php/herpetotropicos/article/view/680/634 
Lötters S. 2007. The fate of the harlequin toads-help through a synchronous multi-disciplinary approach and the IUCN. Amphibian conservation action plan? Mitt Mus Nat kd Berl Zool Reihe. 83: 69-73.

Luger M, Garner TWJ, Ernst R, Hödl W, Lötters S. 2008. No evidence for precipitous declines of harlequin frogs (Atelopus) in the Guyanas. Stud Neotrop Fauna E. 43 (3): 177-80.

Rivera CM. 2005. Atelopus spurrelli. In: Rueda-Almonacid JV, Rodríguez-Mahecha JV, La Marca E, Lötters S, Kahn T, Angulo A (eds.). Ranas arlequines. Bogotá: Conservación Internacional. $158 \mathrm{pp}$.

Rueda LA. 2008. Colorful harlequin frog re-discovered in Colombia. [Revista en línea]. Froglog. 86: 1-2. (Acceso 10 de junio de 2010. URL disponible en: https:// www.amphibians.org/wp-content/uploads/2011/08/ Froglog86.pdf

Schwarz CJ. Estimating power/simple size using Program
Monitor. (En línea) 2013 (Acceso 20 de noviembre). URL http://people.stat.sfu.ca/ cschwarz/Stat-650/Notes/ PDFbigbook-R/R-part020.pdf

Thomas L, Buckland ST, Rexstad EA, Laake JL, Strindberg S, Hedley SL, et al. 2010. Distance software: design and analysis of distance sampling surveys for estimating population size. J Appl Ecol. 47: 5-14.

UICN (Unión Internacional para la Conservación de la Naturaleza). 2013. The IUCN Red List of Threatened Species. Version 2013.2. (Acceso 15 de diciembre de 2013). En: http://www.iucnredlist.org

Vásquez VH, Serrano MA. 2009. Las áreas naturales protegidas de Colombia. Bogotá: Conservación Internacional, Colombia y Fundación Biocolombia.

Young BE, Lips KR, Reaser JK, Ibáñez R, Salas AW, Cedeño JR, et al. 2001. Population declines and priorities for amphibian conservation in Latin America. Conserv Biol. 15 (5): 1213-23. 